\title{
A preliminary analysis of antineoplastic activity of par- vovirus MVMp NS-1 proteins
}

\author{
SHEN Xi ZhONG", ${ }^{*}$, HSIAO CHIEN TSUNG**, YiU WeN \\ YANG*, QING HUA YANG*, SHU DoNG XIAO* \\ * Shanghai Institute of Digestive Diseases, Ren Ji Hos \\ pital, Shanghai Second Medical University, Shanghai \\ 200001, China \\ ** Shanghai Institute of Cell biology, Chinese Academy of \\ Sciences, Shanghai 200031, China
}

\begin{abstract}
Human gastric cancer MKN-45 cells were transfected with pULB 3238, a plasmid carrying MVMp NS-1 gene with its original $\mathrm{P} 4$ promoter replaced by the glucocorticoid inducible promoter MMTV- LTR. After the integration and expression of NS-1 gene, some of the transfectants died, while others remained alive, but the growth features of survived cells were changed. For further study on the antineoplastic function of parvoviral NS-1 protein in vivo, transgenic mice carrying NS-1 genes were established by conventional method. Among 4 founders, one of them was found to be able to transmit the transgene to around 50\% of their offsprings. RT-PCR was performed to indicate the expression of NS-1 gene in transgenic mice and its mRNA appeared in a variety of tissues. The expression of integrated NS-1 gene may correlate with the decreased incidence of tumor induced in vivo by chemical carcinogens.
\end{abstract}

Key words: Autonomous parvovirus $M V M p$, nonstructural proteins, gene expression, transgenic mice, antineoplastic effect.

\section{INTRODUCTION}

1. Corresponding author, Present address: Department of Gastroenterology, Zhong Shan Hospital, Shanghai Medical University, Shanghai 200032, China. 
Parvoviruses are small single-stranded DNA viruses which have been isolated from various animal species, including human being[1]. The low genetic complexity of these viruses may account for their tight dependence on the exogenous factors for their replication which can be provided by helper viruses and/or host cells[2, 3]. The autonomous parvoviral regulatory proteins, designed as NS(nonstructural) proteins, play a central role in the viral life cycle. They regulate the transcription of parvoviral promoters both positively and negatively[4-6] and are absolutely required for parvoviral DNA replication[7, 8]. The NS proteins of parvoviruses also appear to affect many other biological processes, including gene expression programmed by some heterologous promoters[6, 9], initiator-induced DNA amplification[10], in vitro neoplastic transformation[11], stable cell transformation with exogenous DNA[9, 12] etc. The specific mechanisms by which NS proteins inhibit these processes remain poorly understood. Nevertheless, the interference of parvoviral regulatory proteins with DNA synthesis is well documented, unscheduled DNA synthesis initiated at integrated tumor virus sequences can be induced by various carcinogenic treatment, leading to a selective DNA amplification that can be followed at the molecular level[13]. Recent results indicated that the NS proteins of parvovirus minute virus of mice (MVMp) could also inhibit heterologous DNA replication[14], and the intracellular accumulation of NS proteins was shown to result in death of human neoplastic cells[15]. The aim of the present work was to determine whether MVMp NS-1 gene expression was able to interfere with the growth of human gastric cancer cells and whether the expression of NS-1 gene in transgenie mice can inhibit the carcinogenesis or the growth of tumor induced by chemical carcinogens in vivo.

\section{MATERIALS AND METHODS}

\section{Cell line and cell culture}

Human gastric cancer MKN-45 cells were grown in MEM medium Supplemented with $5 \%$ fetal calf serum. The serum was depleted of glucocorticoid hormones by dextran-charcoal treatment[16], in order to maintain the basal level of expression of the glucocorticoid-inducible promoter (see below) as low as possible.

\section{Plasmid and probe}

Plasmid pSV2neo, carrying the aminoglycoside phosphotransferase gene as a selective marker was co-transfected MKN-45 cells with a plasmid, pULB 3238, carrying MVMp NS-1 gene, with its original $\mathrm{P} 4$ promoter replaced by the glucocorticoid inducible promoter of the mouse mammary tumor virus (MMTV-LTR)[15] (Fig 1). This plasmid was kindly provided by Prof. J. Rommelaere (Department of Molecular Biology, Universite Libre de Bruxelles, Belgium). NS-1 gene probe $(5.7 \mathrm{~kb})$ used for Southern blot and RNA dot blot analysis was obtained from pULB 3238 by Bam H1 digestion.

\section{Transfection}

$1 \times 10^{5} \mathrm{MKN}-45$ cells were plated in 6 -cm petri dishes, incubated for $24 \mathrm{~h}$ and cotransfected with both 5-30 $\mu \mathrm{g}$ pULB 3238 and $1 \mu \mathrm{g}$ pSV2neo according to the calcium phosphate precipitation method. 8 geneticin (G418)-resistant cell clones were isolated and amplified, and out of which, 2 
were found to be integrated with pULB3238. RNA dot blot showed that NS-1 gene expression in the transfected cells could be induced by dexamethasone.

Fig 1. Genetic map of the plasmid pULB 3238 (After Caillet-Fauquet P, References [15]).

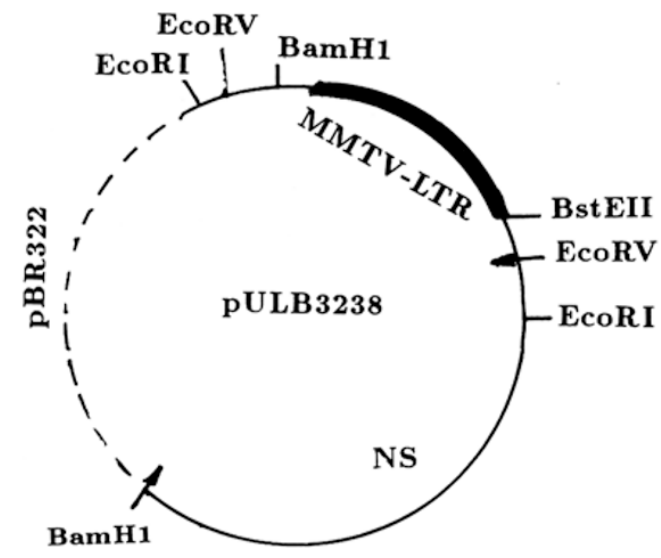

\section{Cell cloning ability on plastic dish}

For the determination of cloning efficiencies on plastic dish, NS-1- transfectants were harvested by trypsinization and replated onto $60-\mathrm{mm}$ dishes (500 cells/dish). Dexamethasone was used at a final concentration of $10^{-4} \mathrm{M}, 10^{-5} \mathrm{M}, 10^{-6} \mathrm{M}$, respectively. After $9 \mathrm{~d}$ of incubation in medium, cultures were fixed in methanol/acetic acid (3:1, v/v) and stained with Giemsa, and the colonies enumerated.

\section{In vivo transplantation studies}

$\mathrm{BALB} / \mathrm{C}-\mathrm{nu} / \mathrm{nu}$ nude mice were maintained in germ-free environment. For cell implantation, five 6-wk-old female nude mice were each given an injection of $3 \times 10^{6}$ control MKN-45 cells and NS-l-transfected MKN-45 cells in the right and left axilla respectively. All the animals were sacrificed at the $14 \mathrm{~d}$ of the experiments and checked for the presence of tumors.

\section{Production of transgenic mice}

DNA microinjection and eggs transfer: After digestion of plasmid pULB 3238 with Bam H1, MVMp NS-1 gene was microinjected into the male pronuclei of fertilized ova of C57BL/SJL F1 mice and the viable eggs following microinjection, as determined by gross morphology, were implanted into the Fallopian tube of pseudopregnant BALB/C foster mothers.

Screening of the potential transgenic mice: PCR and Southern blot were performed to confirm the presence of the NS-1 gene in tail DNA of newborn pups. A 908-base pair fragment encompassing the MVMp NS-1 exon region was amplified by PCR (5'-TGATGGCTCAACCAGGTGGA-3', 5'TGGTGTGCTCCAAGGCTCTA -3')[17]. PCR was performed according to standard protocols given by Promega PCR Kit: thirty-five cycles of amplification were performed, each cycle consisting of denaturation $\left(95{ }^{\circ} \mathrm{C}\right)$, annealing $\left(56{ }^{\circ} \mathrm{C}\right)$ and extension $\left(72{ }^{\circ} \mathrm{C}\right)$ for 45,45 and 90 seconds respectively. $10 \mu \mathrm{l}$ of the PCR products were analyzed by ethidium bromide gel electrophoresis in $1 \%$ agarose, transferred to nitrocellulose filters, and hybridized overnight to ${ }^{32} \mathrm{P}$-labelled probes in the presence of $10 \%$ dextran sulfate, $40 \%$ formamide, $4 \times \mathrm{SSC}, 1 \times$ Denhart's solution, and herring sperm DNA $10 \mu \mathrm{g} / \mathrm{ml}$, and washed twice in $0.1 \%$ SDS in $2 \times$ SSC at room temperature and four times in $0.1 \% \mathrm{SDS}$ in $0.1 \times \mathrm{SSC}$ at $64{ }^{\circ} \mathrm{C}$, and than exposed to XAR film overnight. 
Analysis of antineoplastic activity of parvovirus MVMp NS proteins

\section{Transgene expression}

The expression of NS-1 gene in transgenic mice was investigated in brain, salivary gland, lung, breast, stomach, intestine, liver, kidney, testis and uterus with RT-PCR and agarose electrophoresis, under both dexamethasone-induced and no-dexamethasone condition. Three mice were used in each experiment. Total RNA was first extracted from deep-frozen tissues after homogenization in a $4 M$ guanidine thiocyanate solution as described[18], and cDNA was then synthesized by using $25 \mathrm{U}$ AMV reverse transcriptase (Promega), in a $20 \mu \mathrm{l}$ reaction solution containing $1 \mu \mathrm{g}$ of total RNA

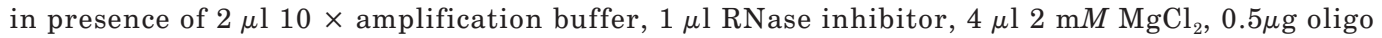
(dT) cellulose 15 primer, and $2 \mu \mathrm{l} 10 \mathrm{mM}$ dNTP mixture. The reaction mixture was incubated for $30 \mathrm{~min}$ at $42{ }^{\circ} \mathrm{C}$, boiled for $5 \mathrm{~min} .5 \mu \mathrm{l}$ of the reverse transcription products were further amplified by PCR and checked by Southern blots as described above.

\section{Chemical induction of tumors}

$0.2 \mathrm{ml}$ of $10 \%$ ethylcarbamate water solution was administered by intraperitoneal injection once a week for 10 times in 41 male offspring of F6 NS-1 transgenic mice. Among them, 19 integrated with NS-1 gene (NS-1(+)), 22 were not integrated with NS-1 gene (NS-1(-)). The mice were sacrificed 30 weeks after first injection and their lung were removed, weighed, and examined for grossly visible lesions. Tumor nodules were scored. After fixation in $10 \%$ formaldehyde solution, each lobe of lung was cut in total into 1.5-mm-thick slices, which were routinely processed for light microscopy. Statistical analysis were made between NS-1 gene(+) and NS-1 gene(-) paired groups for numbers of tumor-bearing mice and average number of tumors per mouse. Methylcholanthrene was used to induce skin cancer. 27 female offspring of F6 NS-1 transgenic mice were used as experimental animal, among them, 14 were NS-1(+), 13 were NS-1(-). $0.2 \mathrm{ml}$ of $0.5 \%$ methylcholanthrene benzol solution was administered by subcutaneous injection at left scapular region, and 2 weeks later the same dose of the carcinogen was administered at the same region of the experimental mice. The mice were observed from the onset of tumor formation (78-102 days) up to the time just before their death, sacrificed, and the average tumor weight, the rate of tumor formed and the prolongation of life were analyzed. Group differences were assessed for statistical significance by using student's $t$ test.

\section{RESULTS AND DISCUSSION}

\section{The characterization of NS-transfected MKN-45 cells}

Human gastric cancer MKN-45 cells were cotransfected with both pULB 3238 and pSV2neo plasmids. Among 8 geneticin-resistant cell clones isolated and amplified, 2 were found to be integrated with NS-1 gene. RNA dot blot showed the NS-1 gene expression in the transfected cells could be induced by dexamethosone (data not shown). After the NS-1 gene was expressed, some of the transfectants died but others remained survived. In comparison with those of MKN-45 controls, the survived NS-1 transfectants had the following characteristics: a. nucleus/cytoplasm ratio reduced; b. generation time prolonged; c. adhesive ability among cells increased; d. cloning efficiency decreased about $40 \%$; and e. formation of tumors in nude mice also suppressed (Fig 2). Histopathologic analysis of tumors formed in nude mice showed that the morphological appearance of NS-1 gene transfected cells was irregular with abundant cytoplasm, relatively low nuclear/cytoplasm ratios and presence of cell necrosis (Fig 3). The mechanisms of cytopathogenecity caused by paroviruses remained elusive. Previous studies showed that the sensitization of a 
number of transformed cells to parvoviral infection was correlated with an increase in their ability to viral DNA replication and/or viral genes expression, in particular, the NS proteins[19]. Caillet-Fauquet et al provided direct evidence that the intracellular accumulation of NS-1 proteins was shown to result in the death of human neoplastic cells[15]. The present study confirmed that NS-1 gene transfection and expression were able to kill and disturb the growth of human gastric cancer cells. The mechanism of action by which NS-1 proteins perturb target cells is presently unknown. Possibly, the target cells might undergo an internal disintegration triggered by a variety of processes, including interruption of host protein synthesis and perturbation of signaling pathways[20].

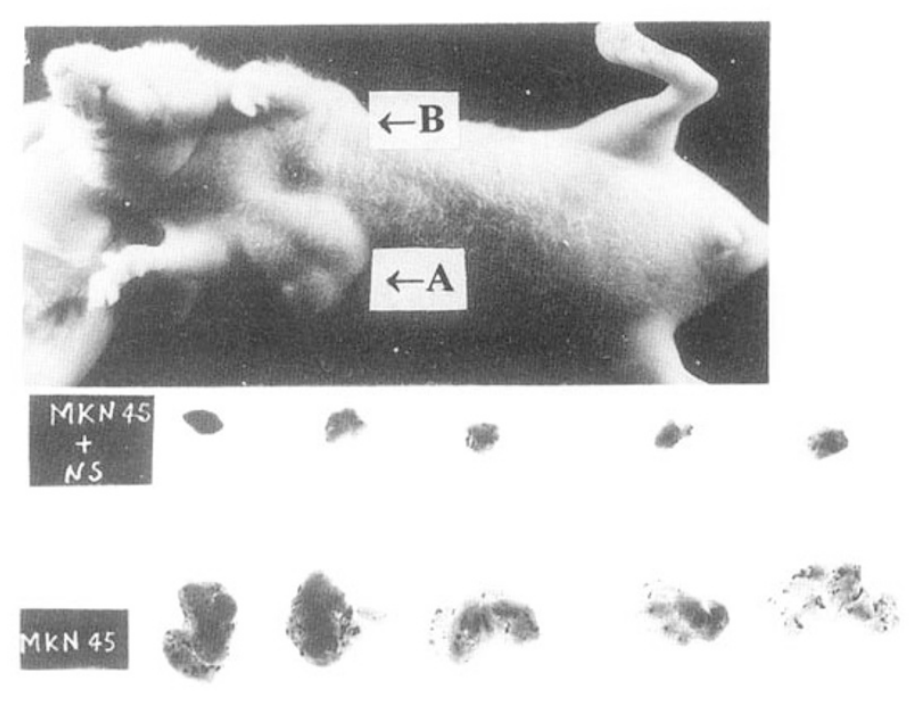

Fig 2. The formation of tumors in nude mice after the inoculation of NS-1 gene transfected MKN45 cells and control MKN45 cells.

A. MKN45: Average tumor weight was $0.885 \pm 0.105 \mathrm{~g}$

B. MKN45+NS: Average tumor weight was $0.115 \pm 0.021 \mathrm{~g}$

\section{The generation of transgenic mice with NS-1 gene}

To examine the biological consequences of NS-1 gene integration in vivo, DNA containing the gene of MMTV-LTR/NS-1 was microinjected into the male pronucleus of fertilized, single cell embryo of C57BL/SJL F1 mice. The 225 eggs so injected were transferred into 9 pseudopregnant foster females (BALB/C). 4 out of 9 foster mothers delivered 15 viable pups (founders, named as F1 to F15 respectively). DNA was then extracted from tails of 10 of the 15 pups. PCR and Southern blot were performed to confirm the presence of the NS-1 gene and 4 founder animals (F2, F6, F13, F14) were found that have retained the transgene[21]. Mating of these trans- 
genic mice to uninjected control C57BL/6J mice has shown that in all 4 founders the NS-1 gene could be transmitted to their offsprings in first generation, and in the case of F6, the rate of transmission was found to be around 50\% (41/84) of their offspring (Fig 4).
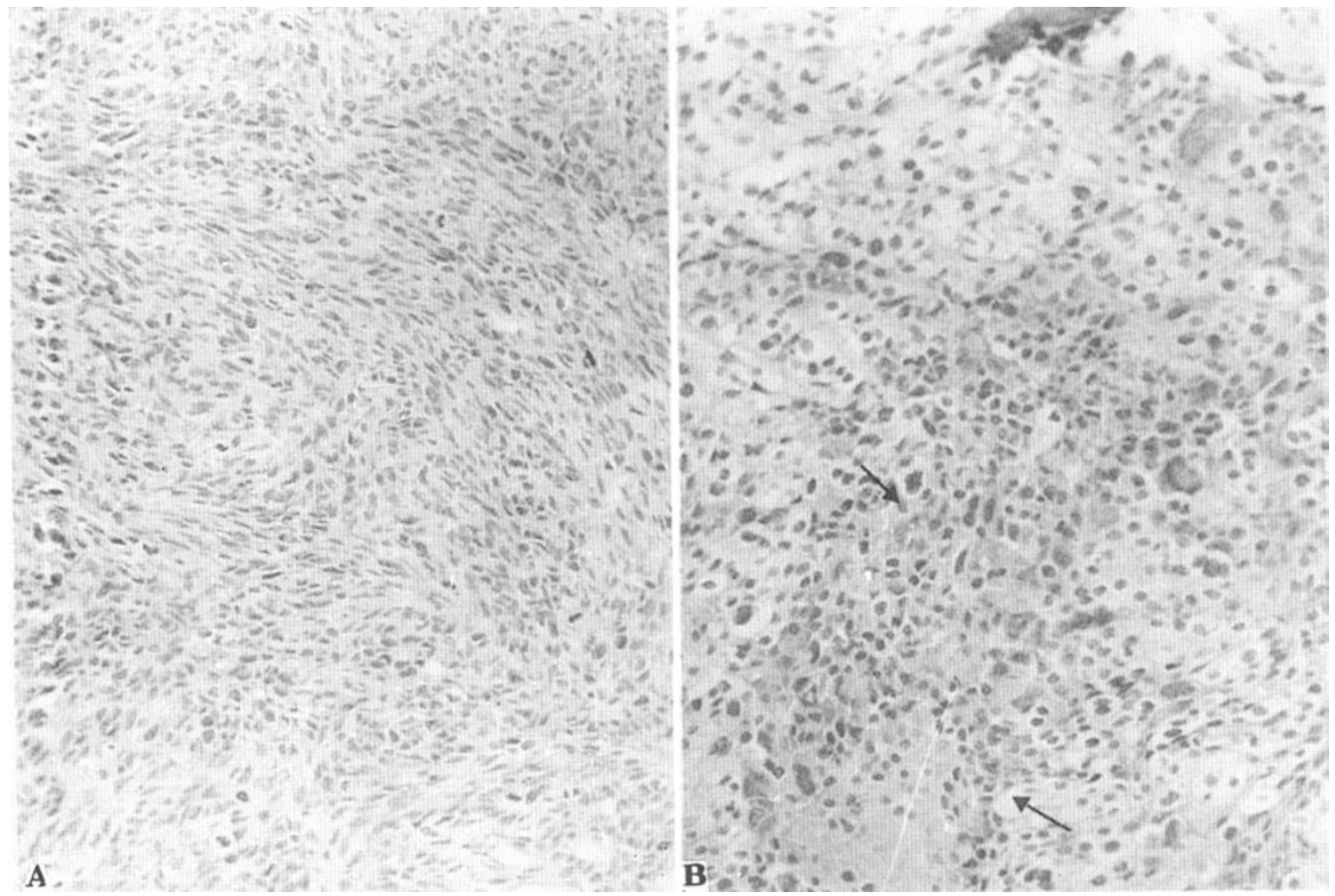

Fig 3. The histological feature of tumor tissue formed in nude mice. A: Control MKN45 cells B: NS-1 gene transfected MKN45 cells (arrow: necrotic area, HE staining, $\times 250$ )

\section{The expression of the integrated NS-1 gene}

It is important to determine the extent of expression of the foreign gene in various organs. The expression of integrated NS-1 gene was investigated in brain, salivary gland, lung, breast, stomach, intestine, liver, kidney, testis and uterus with RTPCR. Without dexamethasone-induction, the mRNA of NS-1 gene appeared in the salivary gland and lung of the offspring F6, but only in the breast of offspring F13. In presence of dexamethasone-induetion, the mRNA of NS-1 gene was present in the tissues of brain, salivary gland, lung, stomach, intestine, kidney and uterus of an offspring from F6 (Fig 5) and was also present in breast, lung and salivary gland of an offspring from F13 (Fig 6). This tissue expression pattern of NS-1 gene was 
Shen XZ et al.

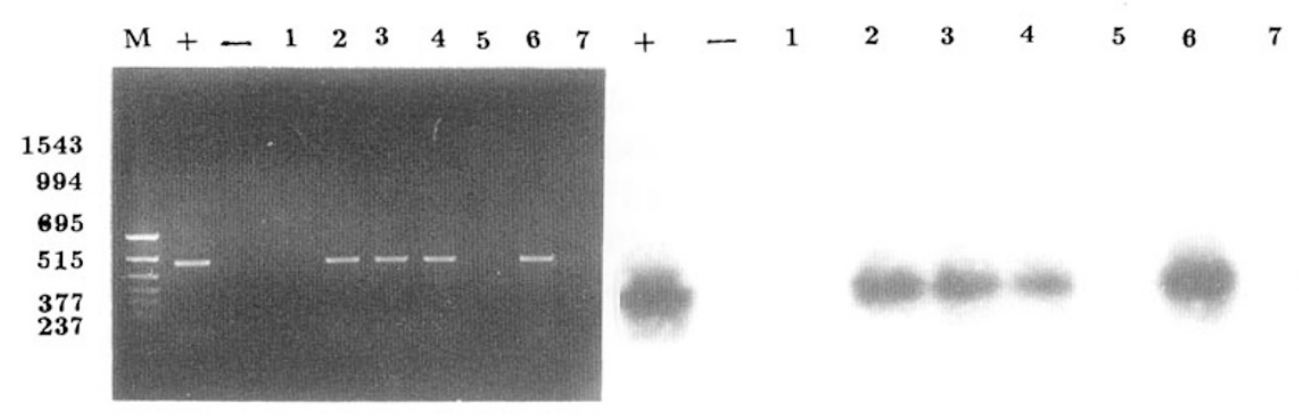

Fig 4. PCR of some F6 offsprings tail DNA and checked by Southern blot(right part)M: PCR marker. +: positive control. - - : negative control. 1-7: offsprings of F6XC57BL/6J mouse.

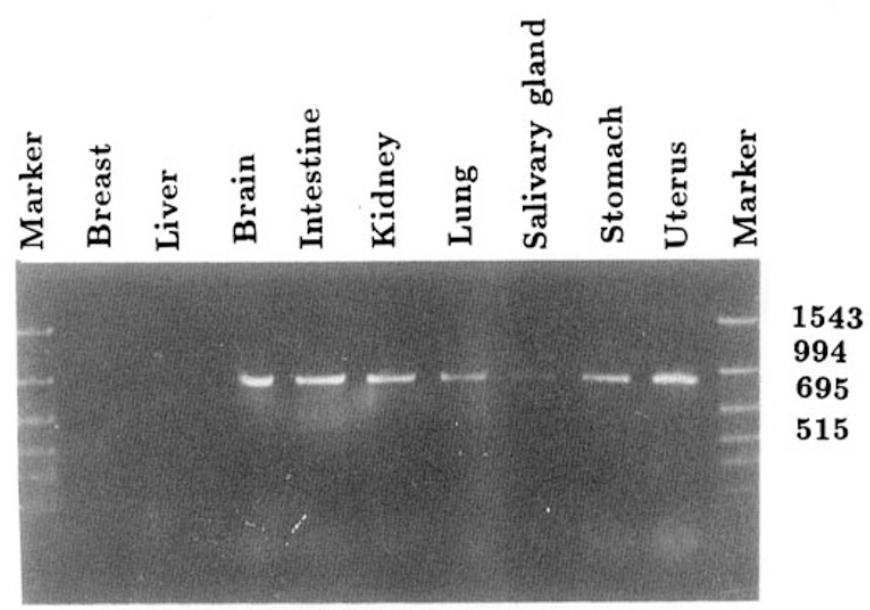

908

Fig 5. Expression of integrated NS-1 gene in the organs of one of F6 offsprings underdexamethasone induced condition, RT-PCR and checked by Southern blot (lower part).

same in other two F6 and two F13 mice. Accounting for the anomalous expression of the MMTV LTR controlled gene remains difficult. Leder et al constructed ten 
Analysis of antineoplastic activity of parvovirus MVMp NS proteins

transgenic mouse strains, each of which carries a form of the MMTV/c-myc fusion gene. Among majority of these strains, expression of fusion gene is restricted only to the breast, salivary gland, and, occasionally the testis and lung[22]. This difference in expression must then be a function of the sequences that surround the site of integration of the transgene or of alterations in the structure of the transgene that occur as a consequence of its integration. Possible mechanisms, such as linkage of the transgene to a constitutively activated control sequence, the creation of a constitutive regulatory mutation, or the loss of a negative control region, deserve consideration. In present study, there was no obvious pathological changes in the tissues which expressed NS-1 gene.

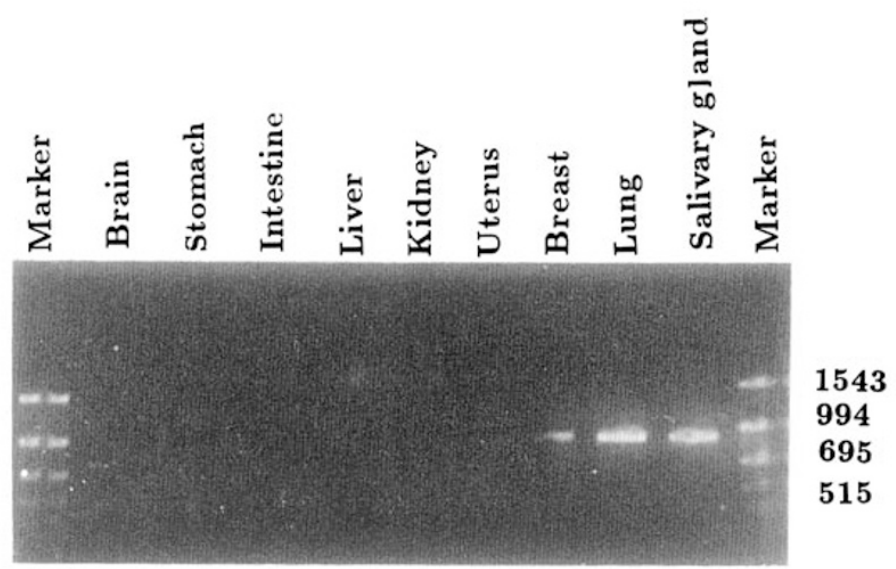

908

Fig 6. Expression of integrated NS-1 gene in the organs of one of F13 offsprings underdexamethasone induced condition, RT-PCR and checked by Southern blot (lower part).

Inhibition of formation of chemical-induced tumors by expression of NS gene

Two chemical carcinogens, ethylcarbamate and methylcholanthrene, were chosen to induce lung (with NS-1 gene expression in this tissue) and skin (without NS-1 gene expression in this tissue) tumor, respectively. Ethylcarbamate water solution was administered by intraperitoneal injection in 41 (19 NS-1(+), $22 \mathrm{NS}-1(-))$ male offspring of F6, and the 36 mice survived during the experiment (17 NS-1(+), 19 NS-1(-)) were killed 30 weeks after first injection and the lung tumor nodules were counted. PCR and RT- PCR confirmed that there were NS-1 gene integration and expression in lung tissues of NS-1(+) group. The result suggested the average num- 
ber of lung tumor nodules in NS-1(+) group were significantly fewer than those in NS-1(-) group $(\mathrm{P}<0.01)$ (Tab 1). Methylcholanthrene benzol solution was administered by subcutaneous injection at left scapular region in 27 (14 NS-1(+), 13 NS-1(-)) female offspring of F6. Except three mice died before the tumor formation. PCR and RT-PCR showed that there was no NS-1 gene expression in skin tissues although this tissue integrated with NS-1 gene in NS(+) group. The results indicated that there appeared no significant difference between NS-1(+) and NS-1(-) group as far as the average tumor weight, rate of tumor formed and prolongation of life were concerned (Tab 2).

Tab 1. Ethylcarbamate induced lung tumors in $\mathrm{F} 6$ offsprings

\begin{tabular}{lcccc}
\hline group & $\begin{array}{l}\text { No. of animals } \\
\text { with tumor }\end{array}$ & $\begin{array}{l}\text { No. of animals } \\
\text { used }\end{array}$ & $\begin{array}{l}\text { Average of tumor } \\
\text { nodules per mouse }\end{array}$ & t test \\
\hline NS-1(-) & $19 / 19$ & $13.74 \pm 2.35$ & \\
NS-1 $(+)$ & $17 / 17$ & $9.96 \pm 1.69$ & $\mathrm{p}<0.01$ \\
\hline
\end{tabular}

Tab 2. Methylcholanthrene induced skin cancer in F6 offsprings

\begin{tabular}{|c|c|c|c|c|}
\hline group & $\begin{array}{l}\text { No. of animals } \\
\text { used }\end{array}$ & $\begin{array}{l}\text { Average tumor } \\
\text { weight }(\mathrm{g})\end{array}$ & $\begin{array}{l}\text { Average prolongation } \\
\text { of life(day) }\end{array}$ & $\mathrm{t}$ test \\
\hline NS-1(-) & $11 / 11$ & $5.36 \pm 1.02$ & 106.911 .5 & \\
\hline $\mathrm{NS}-1(+)$ & $13 / 13$ & $5.40 \pm 1.21$ & 104.312 .7 & $\mathrm{p}>0.05$ \\
\hline
\end{tabular}

* The expression of NS-1 gene in skin by RT-PCR was tested with negtive result (see txet).

The mechanism of parvovirus inhibition of tumorigenesis remains unknown. The evidence accumulated in recent years suggested that oncosuppression by parvoviruses might be mediated, at least in part, by NS proteins [23, 24]. In the present study, application of transgenic mouse technology allowed direct determination of whether the expression of NS-1 gene in mice will render them resistant to tumorigenesis and/or growth inhibition of tumor induced by carcinogens. We found a statistically significant reduction in the number of lung tumor nodules in transgenic mice in paired groups which received ethylcarbamate treatment. In our experiment, the copy number of NS-1 gene integrated is low (unpublished data), otherwise the reduction of lung tumor nodule formation would have been more prominent. Contrary to the results of ethylcarbamate induction of lung tumor, the difference of average tumor weight, the rate of tumor formed and prolongation of life were not significant between NS-1(+) group and NS-1(-) group following their methylcholanthrene treatment, a fact which may be related to the non-expression of NS-1 gene in skin tissues. Initially, we have not included skin tissue in our RT-PCR analysis. But later in skin tumor induction by methycholanthrene, we arbitrarily took the skin tissue of some NS-1 gene(+) experimental animals, both before the application of methycholanthrene and at end of the experiment, to study NS-1 gene expression by RT-PCR. 
The results were all negative in these determinations. Therefore, we consider that our data provide direct evidence that only the NS-1 proteins can inhibit the formation of tumor induced by chemical carcinogens. Although we cannot completely preclude the possibility that exogenous gene integration in transgenic experiments may affect the genetic background, thus influencing tumor induction. However, we could not find any gross abnormality in our transgenic mice. As mentioned in the introduction of the present paper, NS-1 proteins appear to interfere with many important biological processes other than parvoviral DNA replication, NS-1 protein may impinge upon the cell ability to initiate DNA synthesis, or may down-modulate the expression of some cellular genes, in particular oncogenes, thereby preventing cellular proliferation. Mutational analyses of DNA clones from several autonomous parvoviruses indicate that NS-1 expression impairs various cellular responses through a presumably cytotoxic mechanism[25]. Whether the NS- 1 proteins of autonomous parvoviruses can also suppress transformation without killing effect is presently unclear. Our results seem to lend support to this possibility.

\section{ACKNOWLEDGMENTS}

We thank Dr J. Rommelaere for plasmid pULB 3238, Professor Luo ZY for plasmid pSV2neo. We are indebted to Xu GJ for help in maintaining lines of transgenic mice. This work was supported by National Natural Science Foundation of China (NO. 39270764, NO. 39570782).

\section{REFERENCES}

[1] Anderson MJ. Parvoviruses as agents of human disease. Prog Med Virol 1987; 34:55-69.

[2] Berns KI, Bohenzky RA. Adeno-associated viruses: an update. Adv Virus Res 1987; 32:243-307.

[3] Cotmore SF, Tattersall P. DNA replication in the autonomous parvovirus. Semin Virol 1995; 6:271-81.

[4] Beaton A, Palumbo P, Berns KI Expression from the adeno-associated virus p5 and p19 promoters is negatively regulated in trans by the rep protein. J Virol 1989; 63:4450-4.

[5] Doerig G, Hirt B, Bearo B et al. Minute virus of mice nonstructural NS-1 protein is necessary and sufficient for transactivation of the viral p38 promoter. J Gen Virol 1988; 69:2563- 73.

[6] Rhode SL, Richard SM. Characterization of the trans-activation-responsive element of the parvovirus HI P38 promoter. J Virol 1987; 61:2808-15.

[7] Hermonat P, Labow MA, Wright $\mathrm{R}$ et al. The genetics of adeno-associated virus isolation and preliminary characterization of mutants. J Virol 1984; 51:329-39.

[8] Merchlinsky M J, Tariersall P J, Leary JJ et al. Construction of an infectious clone of autonomous parvovirus minute virus of mice. J Virol 1983; 47:227-32.

[9] Labow MA, Graf LH, Berns KI Adeno-associated virus gene expression inhibits cellular transformation by heterologous genes. Mol Cell Biol 1987; 7:1320-5.

[10] Heibronn R, Burkle A, Stephan S et al. The adeno-associated rep gene supresses herpes simplex virus-induced DNA amplification. J Virol 1990; 64:3012-8.

[11] Hermonat PL. The adeno-associated virus Rep 78 gene inhibits cellular transformation induced by bovine papillomavirus. Virology 1989; 172:253-61.

[12] Brandenburger A, Legendre D, Avalosse Bet al. NS-1 and NS-2 proteins may act synergistically in the cytopathogenicity of parvovirus MVMp. Virology 1990; 174:576-84. 
[13] Lavi S. Carcinogen-mediated activation of SV40 replications: A model system for indication of carcinogenesis in "Gene Amplification" (R.D.Schimke. Ed.). 1982 pp. 225-30 Cold Spring Harbor laboratory. Cold Spring HarbOr. NY.

[14] Tenenbaum L, Dupont P, Spegelaere Pet al. Inhibition of heterologous DNA replication by the MVMp nonstructural NS-1 protein: identification of a target sequence. Virology 1993; 197:630-41.

[15] Caillet-Fauquet P, Perros M, Brandenburger A et al. Programmed killing of human cells by means of an inducible clone of parvoviral genes encoding non-structural proteins. EMBO J 1990; 9:2989-95.

[16] Stanley ER, Palmer RE, Sohn U. Development of methods for the quantitative in vitro analysis of androgen-dependent and autonomous shionogi carcinoma 115 cells. Cell 1977; 10:35-44.

[17] Huang QS, Huang J, Luo ZY. Cloning of parvovirus H1 NS partial gene by PCR. Virologica Sinica 1995; 10:323-31.

[18] Chomczynski P, Sacchi N. Single-step method of RNA isolation by acid guanidinium thiocyanatephenol-chloroform extraction. Analytic Biochemistry 1987; 162:156-9.

[19] Van Hille B, Duponchel N, Salome N et al. Limitations to the expression of parvoviral nonstructural proteins may determine the extent of sensitization of EJ-ras-transformed rat cells to minute virus of mice. Virology 1989; 171:89-97.

[20] Shen XZ, Yu GH, Jiang SJ et al. The effects of parvoviral nonstructural proteins gene transfection on the expression of cytokines in gastric cancer cell. Chin J Biother 1996; 3:203-6.

[21] Shen XZ, Cong XQ, Jiang SJ et al. Preparation of parvoviral nonstructural protein gene transgenic mice. Chin J Cell Biology 1996; 18:127-30.

[22] Leder A, Pattengale PK, Kuo A et al. Consequences of widespread deregulation of the c-myc gene in transgenic mice: multiple neoplasms and normal development. Cell 1986; 45:485-95.

[23] Vanacker JM, Rommelaere J. Non-structural proteins of autonomous parvoviruses: from cellular effects to molecular mechanisms. Semin Virol 1995; 6:291-7.

[24] Rommelaere J, Cornelis JJ. Antineoplasic activity of parvovirus. 3 Virol Methods 1991; 33:23351.

[25] Jacoby RO, Ball-Gordrich LJ. Parvovirus infections of mice and rats. Semin Virol 1995; 6:32937.

\section{Received 21-7-1997. $\quad$ Revised 17-11-1997. Accepted 17-11-1997}

